AC 2007-2211: A COST-EFFECTIVE AUTOMATION AND ROBOTICS LAB

John Anderson, Oregon Institute of Technology 


\title{
A Cost Effective Automation \& Robotics Lab
}

\begin{abstract}
Much work has been published in the area of design of laboratory exercises and facilities to support teaching robotics and automation. New opportunities are becoming available, however, to allow laboratory facilities that are portable, have applications to a wide range of subjects, and are inexpensive.

A large software manufacturer has recently entered the robotics software arena with a unique approach. They are supporting small hobby class robots as well as larger industrial units. This provides an opportunity to build small robot models that may easily be scaled to large industrial systems. This paper describes the construction of small 6 axis robot manipulators and the development of lecture and laboratory components to capitalize on them. Materials were developed in the areas of kinematics, dynamics, control and interfacing with work cells, integration with industrial controls, and programming. Development of industrial scale systems using the same software architecture is also addressed.
\end{abstract}

\section{Introduction}

This paper describes an approach to teaching a robotics and automation course in an undergraduate engineering curriculum.

Traditionally the undergraduate course in robotics is organized in one of two forms. These are;

- Kinematics of manipulators

- Programming and system integration of robots

Typically ABET EAC engineering programs focus on the kinematics, and ABET TAC engineering technology programs focus on system integration and programming. Both typically include some material on characterizing robots by their work envelope, i.e., cylindrical, rectangular coordinate, etc.

Both of these approaches are valid for undergraduate engineering students, but it is difficult to fit all of the material in a one semester course, much less a one quarter course. Since these courses are generally elective courses, it is very difficult to fit a two course sequence into most programs.

The approach at Oregon Institute of Technology has been to offer a senior level course which examines the selection and programming of robots as well as the selection and design of the material handling and fixed automation devices that comprise an automated work cell. One of the problems with this approach is that student do not get to spend much time on the design of the fixed automation pieces. These design parameters are similar to that of the robot, so if additional attention is spent in this area it would serve two purposes.

A problem with this approach is that industrial robots are expensive, and much of the detailed design data on the robot is held as company proprietary. Allowing student to disassemble and examine the structure of a robot costing $\$ 15,000$ to $\$ 20,000$ did not seem to be a good approach, particularly when you would need multiple robots to accommodate a lab section of 15 students. 
There are some hobby robots available, but they typically have low payloads and the controls are not consistent with those of industrial robots. Moreover, the focus of most of the hobby class of robots is on mobile robots, and not on the types most often encountered in manufacturing enterprise.

A new opportunity has arisen with the entry of the Microsoft Corporation into the robot software marketplace with Microsoft Robotics Studio. Microsoft has taken an open architecture approach to the development of their software and has targeted the hobby and education markets initially to build a user base and to support software development. This allows the development of user interfaces for small inexpensive robots and fixed automation that is consistent with the user interface approaches of major suppliers of industrial robots.

Oregon Institute of Technology is also a university affiliate for Project Lead the Way (PLTW). PLTW is a pre-engineering program aimed at high schools and one module of the program is Computer Integrated Manufacturing (CIM). Currently the approved robots for the CIM module run about $\$ 10,000$ to $\$ 15,000$. One of the goals of Oregon Institute of Technology is to develop equipment which will allow high schools in the region to participate in the CIM program at a lower initial cost.

\section{Robot}

Lynxmotion, Inc. ${ }^{1}$ specializes in small robots for the hobby and education market. They offer kits and assembled robots, as well as parts and accessories. While most of their products serve the mobile hobby robot market they offer a six axis robot manipulator

Their robots are based on servo motors designed for use in radio control models. These servo motors contain the motor, gearbox, position feedback mechanism, and control electronics ${ }^{2}$. They are controlled by a DC pulse of approximately 5 volts amplitude, with a pulse width from 1 to 2 $\mathrm{ms}$. The width of the pulse determines the angular position of the servo. The servos typically have $180^{\circ}$ angular range and are available in torques from 57 in oz up to 333 in oz. Weights vary from 1.5 to $2 \mathrm{oz}$.

Lynxmotion offers a complete six axis robot kit, the Lynx 6. This robot is designed with links constructed of flat Lexan panels with push in plastic pins for assembly. This makes for an easy to assemble robot, but the finished product is not very robust.

The company also offers a series of components referred to as the Servo Erector Set. This is not a defined kit of parts, but rather a series of motor mounts and brackets designed to work together, which can be used to assemble a variety of robot configurations. A four axis robot arm assembled with these components is shown in Figure 1.

The use of this kit allows interchangeability of servo motors, thus allowing for larger payloads. The links are based on 6061 T6 aluminum tubing with standard lengths from 1.5 " to 6".

Another interesting feature of the servo erector set is the ability to easily model the arm components in a CAD system. The link shown in Figure 2 is an assembly model designed in Autodesk® Inventor. Lynxmotion has a library of parts files in Solidworks and Step formats. 


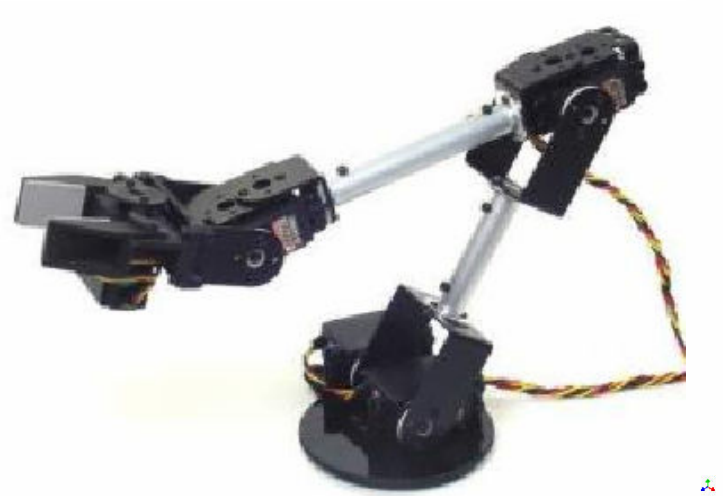

Figure 1. Four DOF robot arm

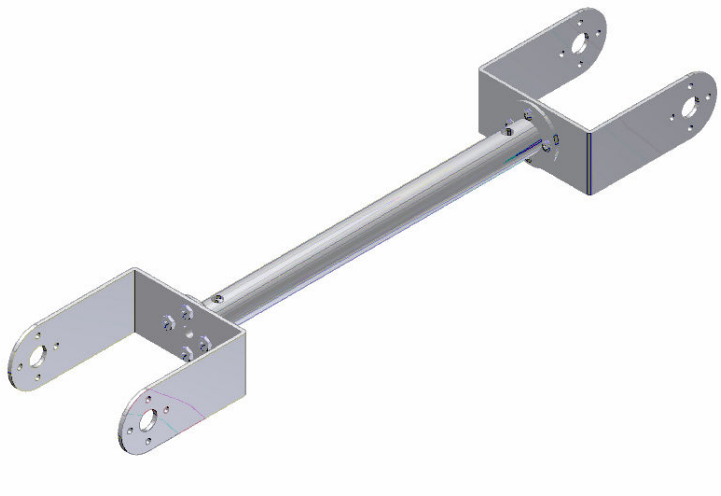

Figure 2. Assembly model of link

The components necessary to build a six axis robot with these components costs approximately $\$ 690$. This cost is based on the use of $168 \mathrm{oz}$ in servos. Use of the most powerful servos, the 333 oz in servo, would increase the overall cost by approximately $\$ 400$, and would significantly increase the pay load of the robot.

\section{Software}

Lynxmotion markets a Windows ${ }^{\circledR}$ based robot control program named RIOS (Robotic arm Interactive Operating System). The program is very flexible and will effectively control the robot, but the operator interface is not consistent with that of commercial industrial robots. It has the capability to program a series of points and a path for the robot to take, but it is not very user friendly.

Microsoft ${ }^{\circledR}$ Corporation has entered the robot software market with a product titled MS Robotics Suite ${ }^{3}$. It is based on the idea of high level control of a number of "services". These services are either hardware or software devices. For example, the Lynxmotion SSC 32 servo controller board is defined as a service. There is also an inverse kinematics software engine included with the software that is defined as a service. 


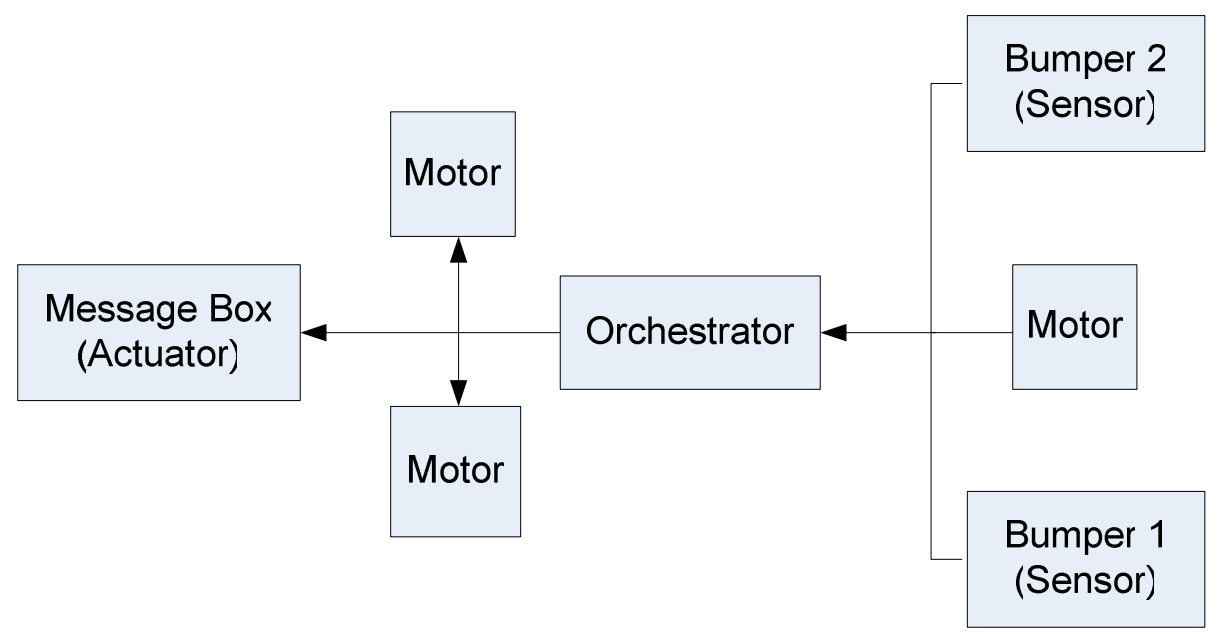

Figure 3. MS Robotics Suite robotics application with three sensors and three actuators.

Figure 3 above is a block diagram of the interaction between the software (the "orchestrator") and the physical inputs and outputs. This high level interaction allows many components to be coordinated by a single software system.

In the case of the Lynxmotion robot, Robotics Suite allows one to develop a user interface that is more consistent with commercial robot systems. A student design team is currently building a control system for the Lynxmotion servo kit base robot using this approach. This software will be available to other schools at low or no cost.

MS robotics Suite also includes a visual programming language and a simulation module. MS Robotics Suite is available for personal or educational use at no cost.

\section{Course Content}

The Lynxmotion robots were incorporated into the MFG 453 Automation and Robotics class in the fall of 2006. There were 12 students in this class and it was organized into topic areas as follows;

- Introduction to Automation

- Robot Workspace

- Robot Programming

- Load Analysis

- Kinematics

- Material Handling Devices

In the first two topics students are introduced to a series of industrial robots, applications studies, and to an educational work cell. Students examine the joint structure of existing industrial robots in order to better understand work space and constraints.

The learning objectives for this course were as follows. At the completion of the course the students will be able to

1. Program commercial robots.

2. Design workcells incorporating robots. 
3. Select material handling devices for work cells.

4. Analyze the fundamental economics of varying levels of manufacturing automation.

5. Understand the relationship between product design and product manufacturing at various production levels.

6. Understand the advantages and disadvantages of robotic systems compared to other automation processes.

The educational work cell is a machine loading application with a small CNC mill, a gravity parts feeder, and an Intelitek Scorbot ${ }^{4}$ robot with a one meter linear slide base. This configuration is the one of the approved equipment configurations by PLTW. Students program the robot to perform machine loading and palletizing applications.

Robot programming is primarily done with the Scorbot and the Lynxmotion machines. This gives students an opportunity to explore the workspace constraints of the systems and more fully appreciate joint configurations.

In the load section, lecture topics in static and dynamic load conditions, torque requirements, drive configuration, and link compliance are coupled with labs focused on selecting components, analysis to predict performance, building, and testing of configurations of the Lynxmotion robots.

One of the advantages of using the Lynxmotion machine is that it is simple enough to visualize and obtain physical size and mass data on all the components. Figure 5 below illustrates a portion of the part data collected by the students. This helps develop an appreciation of the impact of component selection on performance.

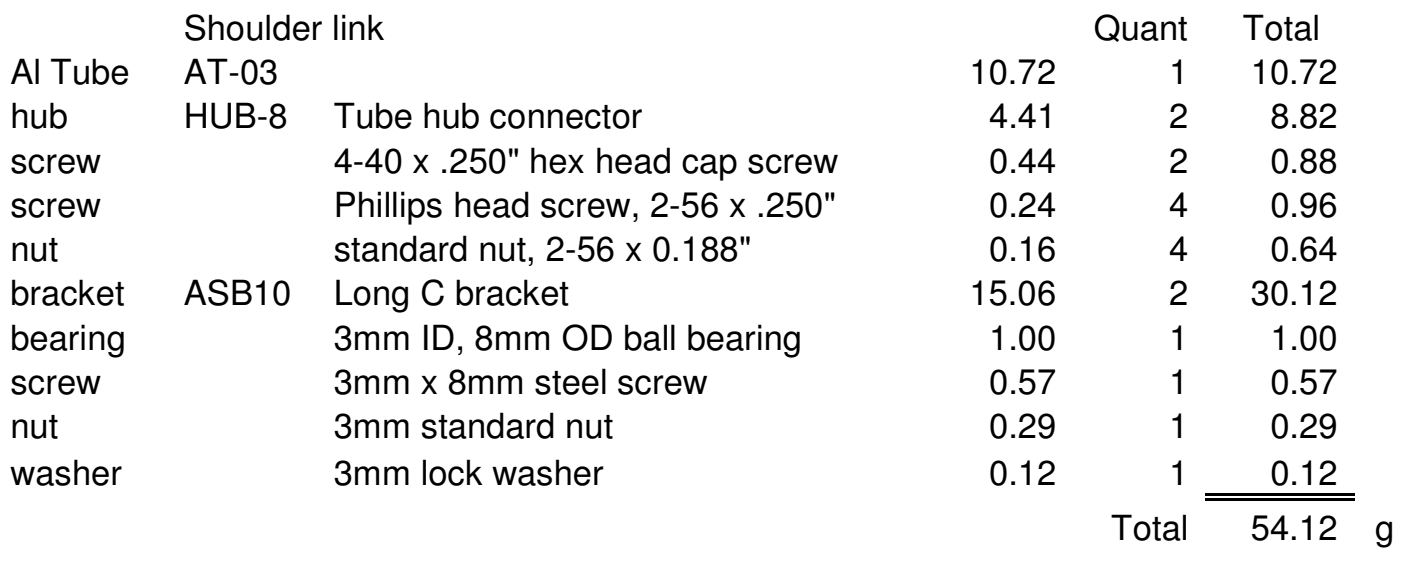

Table 1. Weights of components in Lynxmotion robot link

For the kinematics portion of the course an abbreviated approach was employed in the first offering of the course. The text "Robot Modeling and Kinematics"s was used in the course. The derivation of transformation matrices in a planar model was discussed and then the Denavit Hartenberg modeling technique was introduced. The text offers some excellent visualization and analysis software. This allows an approach that focuses more on applications than the underlying mathematics. 
Finally in the material handling area, lecture topics included conveying systems and parts feeders. Lab work focused on use of vibratory feeders to orient parts for assembly. A small design project was included, where the student was required to design a ramp and selectors to orient and feed a relay.

\section{Future Work}

One of the goals of the development of the Lynxmotion robot with the Robotics Suite controller is to demonstrate a less expensive alternative for PLTW. Part of the recommended apparatus for PLTW is a 6 axis robot with a slide base. The Lynxmotion servo controller board has the capability of controlling additional RC servos, and of providing a binary output. Since the RC servos are controlled by angular positioning using a potentiometer, it will be difficult to adapt them to an application that controls a linear distance using multiple rotations of the motor. On the other hand, a rotary base (turn table) might accomplish the same goal and would be easier to implement. Lynxmotion, in fact, markets a small turn table for this type application.

Simulation of Lynx Robot The MS Robots Suite includes a simulation package. One of the next goals is to model the Lynxmotion robot so that programs may be executed off line.

Oregon Institute of Technology has several old robots from the 1970's that are still mechanically sound, but whose controls are hopelessly out of date. Modern stepper or servo drives are available relatively inexpensively. Software drivers will be written for the some of these devices to allow MS Robotics Suite to address them as services. The software written for the Lynxmotion controls will be modified to allow the same user interface to drive these robots.

The advantage in reusing this old equipment is that all of the detailed design information of the link mechanical and electrical drive will be available to students.

\section{Conclusion}

A robot course that uses relatively low cost equipment has been developed. This allows students to do analysis of robotic systems and then implement and test their designs.

A question on the student evaluation of the course asked student to rank the various sections of the course (see above). $90 \%$ of the respondents indicated the analysis and lab work with the Lynxmotion robots was the most effective portion of the course.

The overall student satisfaction for this course increased $10 \%$ over the same course taught six months earlier by the same faculty member.

\section{Bibliography}

1. Lynxmotion, Inc., http://www.lynxmotion.com/

2. Buiding Robot Drive trains, Dennis Clark \& Michael Owings, 2003, McGraw Hill

3. Microsoft Corporation, http://msdn.microsoft.com/robotics/

4. Intelitek, http://www.intelitek.com/products/product.asp?cid=1\&pid=4\&did=10

5. Manseur, Rachid, Robot Modeling and Kinematics, 2006, Charles River Media 